\title{
A comparative evaluation of coenzyme Q I0-loaded liposomes and solid lipid nanoparticles as dermal antioxidant carriers
}

This article was published in the following Dove Press journal:

International Journal of Nanomedicine

21 September 2012

Number of times this article has been viewed

\author{
Evren H Gokce' \\ Emrah Korkmaz' \\ Sakine Tuncay-Tanrıverdi' \\ Eleonora Dellera ${ }^{2}$ \\ Giuseppina Sandri² \\ M Cristina Bonferoni ${ }^{2}$ \\ Ozgen Ozer'
}

'Department of Pharmaceutical Technology, Faculty of Pharmacy, University of Ege, Izmir, Turkey; ${ }^{2}$ Department of Drug Sciences, University of Pavia, Pavia, Italy
Correspondence: Ozgen Ozer Faculty of Pharmacy, University of Ege, Osman Kibar Meydani, 35I00,

Bornova, Izmir, Turkey

Tel +902323|| $4|8|$

$\mathrm{Fax}+902323885258$

Email ozgen.ozer@ege.edu.tr
Background: The effective delivery of coenzyme Q10 (Q10) to the skin has several benefits in therapy for different skin pathologies. However, the delivery of Q10 to deeper layers of skin is challenging due to low aqueous solubility of Q10. Liposomes and solid lipid nanoparticles (SLN) have many advantages to accomplish the requirements in topical drug delivery. This study aims to evaluate the influence of these nanosystems on the effective delivery of Q10 into the skin.

Methods: Q10-loaded liposomes (LIPO-Q10) and SLNs (SLN-Q10) were prepared by thin film hydration and high shear homogenization methods, respectively. Particle size (PS), polydispersity index (PI), zeta potential (ZP), and drug entrapment efficiency were determined. Differential scanning calorimetry analysis and morphological transmission electron microscopy (TEM) examination were conducted. Biocompatibility/cytotoxicity studies of Q10-loaded nanosystems were performed by means of cell culture (human fibroblasts) under oxidative conditions. The protective effect of formulations against production of reactive oxygen species were comparatively evaluated by cytofluorometry studies.

Results: PS of uniform SLN-Q10 and LIPO-Q10 were determined as $152.4 \pm 7.9 \mathrm{~nm}$ and $301.1 \pm 8.2 \mathrm{~nm}$, respectively. ZPs were $-13.67 \pm 1.32 \mathrm{mV}$ and $-36.6 \pm 0.85 \mathrm{mV}$ in the same order. The drug entrapment efficiency was $15 \%$ higher in SLN systems. TEM studies confirmed the colloidal size. SLN-Q10 and LIPO-Q10 showed biocompatibility towards fibroblasts up to $50 \mu \mathrm{M}$ of Q10, which was determined as suitable for cell proliferation. The mean fluorescence intensity \% depending on ROS production determined in cytofluorometric studies could be listed as Q10 $\geq$ SLN-Q10 > LIPO-Q10.

Conclusion: The LIPO-Q10 system was able to enhance cell proliferation. On the contrary, SLN-Q10 did not show protective effects against ROS accumulation. As a conclusion, liposomes seem to have advantages over SLN in terms of effective delivery of Q10 to skin for antioxidant purposes.

Keywords: solid lipid nanoparticles, liposomes, coenzyme Q10, antioxidant, cytotoxicity

\section{Introduction}

The skin is the body's largest organ with an area of approximately $2 \mathrm{~m}^{2}$. Its main function is to protect the body from external threats such as toxins, pathogens, and UV radiation. After being absorbed by molecules in the skin, UVA initiates the formation of reactive oxygen species (ROS), causing oxidative damage to lipids, proteins, and DNA. ${ }^{1}$ Several endogenous mechanisms also contribute to the production of ROS and excessive amounts of ROS is thought to be a key contributor to some pathologies like cancers. ${ }^{2,3}$ To be able to cope with these oxidative stresses produced by internal and external factors, the skin has both enzymatic (superoxide dismutase, catalase, 
and glutathione peroxidase) and nonenzymatic (antioxidant) mechanisms for protection. ${ }^{1}$

Q10, the only lipid soluble endogenous antioxidant, is a component of the electron transport chain in most eukaryotic cells, primarily in the mitochondria. It has an important role in generating adenosine triphosphate during aerobic cellular respiration and inhibits cell membrane peroxidation in the reduced form. ${ }^{4,5}$ However, to deliver Q10 to deeper layers of skin is challenging due to the low aqueous solubility of Q10 and due to the barrier function of stratum corneum. ${ }^{6}$

As lipidic nanocarriers, liposomes and solid lipid nanoparticles (SLN) have taken the interest of scientists for their potential to overcome the topical drug delivery challenges. ${ }^{7}$ Liposomes are promising delivery systems that accomplish the requirements for a topical drug delivery. Topical liposomes may increase solubilization and act as a local depot for sustained release of dermally active compounds, and can enhance penetration. ${ }^{8}$ SLNs are alternative colloidal carrier systems based on solid lipids. SLNs for dermal applications include many advantages such as occlusion, drug targeting, and modulation of drug release. ${ }^{9}$ SLNs favor drug penetration into the skin and sustain the release to avoid systemic absorption. ${ }^{10}$ Chemically unstable compounds that are sensitive to light, oxidation, and hydrolysis can be protected in the form of SLNs. ${ }^{11,12}$

In light of this knowledge, this study was designed to evaluate the influence of these two lipid based nanosystems on the effective delivery of Q10 into the skin. Q10loaded liposomes (LIPO-Q10) and SLNs were prepared, characterized, and their biocompatibility/cytotoxicity was evaluated by means of cell culture (human fibroblasts) under oxidative conditions. Their antioxidant effects on the production of ROS were comparatively evaluated by cytofluorometry studies.

\section{Materials and methods Materials}

Phosphatidylcholine from soybean (Lipoid S100) was supplied by Lipoid (Ludwigshafen, Germany) and Labrasol ${ }^{\circledR}$ Compritol $^{\circledR} 888$ ATO (glyceryl behenate) was obtained from Gattefossé (Lyon, France). Poloxamer ${ }^{\circledR} 188$ (Pluronic ${ }^{\circledR}$ F68) was donated by BASF (Ludwigshafen, Germany). Cholesterol, Tween ${ }^{\circledR} 80$ (polysorbate 80 ) and Q10 were obtained from Sigma-Aldrich (St Louis, MO). All high-performance liquid chromatography (HPLC) reagents and chloroform were purchased from Sigma-Aldrich. The other chemicals were obtained from Merck KGaA (Darmstadt, Germany). All filters were purchased from Sartorius (Göttingen, Germany).

\section{Preparation of liposomes}

The thin film hydration method was used to prepare the liposomal suspensions. ${ }^{13}$ Phospholipid-Lipoid S100 (25 mg), cholesterol (6.25 mg), and Q10 (5 mg) were dissolved in chloroform $(12.5 \mathrm{~mL})$, and organic solvent was evaporated at $40^{\circ} \mathrm{C}$ to form a thin film (Ika RV 10 rotary evaporator ; IKA ${ }^{\circledR}$ Werke GmbH \& Co., Staufen, Germany). Subsequently, the resulting thin film was hydrated in $25 \mathrm{~mL}$ of bidistilled water by rotating in the same evaporator at $100 \mathrm{rpm}$ without using any vacuum. The liposomal suspension was homogenized using ULTRA-TURRAX ${ }^{\circledR}\left(\right.$ IKA $^{\circledR}$ T25; IKA ${ }^{\circledR}$-Werke) at 3500 rpm.

\section{Preparation of SLNs}

Q10-loaded SLNs (SLN-Q10) were prepared by the high shear homogenization method. ${ }^{14} \mathrm{C} 888$ was employed as the lipid base, Poloxamer 188 (P188) and Tween 80 (Tw 80) were used as surfactant and cosurfactant, respectively. As reported in our previous study, ${ }^{14}$ the lipid phase consisting of $300 \mathrm{mg}$ Compritol $^{\circledR} 888$ ATO (C888) and $5 \mathrm{mg}$ Q10 were mixed and heated to $85^{\circ} \mathrm{C}$. At the same time, the aqueous phase consisting of $150 \mathrm{mg}$ of P188 and $75 \mathrm{mg}$ of Tw 80 in $12.5 \mathrm{~mL}$ bidistilled water was heated to the same temperature. The aqueous phase was poured into the lipid phase drop by drop and mixed with ULTRA-TURRAX (T25) at a speed of 24,000 rpm. Then the pre-emulsion produced was dispersed in $12.5 \mathrm{~mL}$ bidistilled water at $4^{\circ} \mathrm{C}$ and kept at $-20^{\circ} \mathrm{C}$ for 10 minutes to obtain nanoparticles by solidification. Blank SLNs were prepared in a similar way, without addition of Q10.

\section{Measurement of particle size (PS) and polydispersity index (PI)}

Liposomes and SLN dispersions were characterized in terms of PS and PI at $25^{\circ} \mathrm{C}$ by photon correlation spectroscopy (Zetasizer-Nano ZS; Malvern Instruments Ltd, Malvern, UK) at an angle of $173^{\circ}$. The formulations were diluted with bidistilled and filtered $(0.45 \mu \mathrm{m})$ water before the experiment $(n=6)$. Each sample was measured in triplicate.

\section{Measurement of zeta potential (ZP)}

The ZP of liposomes and SLN dispersions was measured at $25^{\circ} \mathrm{C}$, under an electrical field of $40 \mathrm{~V} / \mathrm{cm}$ (Zetasizer-Nano ZS). The measurements were conducted in triplicate.

\section{Drug entrapment efficiency}

SLN dispersions or liposomes were placed in a dialysis bags (cutoff $25 \mathrm{kDa}$ ). These bags were centrifuged for 1 hour at 
$14,000 \mathrm{rpm}$ in a medium consisting of 5\% of Labrasol. Then the amount of Q10 in 5\% Labrasol solution was analyzed by a validated HPLC method and the quantity of free drug was calculated. The encapsulated amount of Q10 was calculated by subtracting the free amount of Q10 from the total amount in the dispersion. Each batch was evaluated three times. The following equation was used to calculate the entrapment efficiency (EE\%), where $W_{i}$ is the amount of initial drug and $W_{f}$ is the amount of free drug:

$$
E E \%=\frac{W_{i}-W_{f}}{W_{i}} \times 100
$$

The HPLC system (Agilent Series 1100) consisted of a C18 reverse phase column (ACE 5-C18 $250 \mathrm{~mm} \times 4.6 \mathrm{~mm}$ ). The mobile phase was a mixture of 65:35 methanol: $n$-hexan $(\mathrm{v} / \mathrm{v}) .{ }^{15}$ The flow rate and UV wavelength were set at $1 \mathrm{~mL} /$ minute and $275 \mathrm{~nm}$, respectively.

\section{TEM (transmission electron microscopy) analysis}

TEM (CM12 Philips; Philips, Amsterdam, The Netherlands) was used for the morphological examination of liposomes and SLNs. $2 \%(\mathrm{w} / \mathrm{v})$ phosphotungistic acid was used to stain the samples and after staining they were placed on copper grids for viewing.

\section{Differential scanning calorimetry (DSC) analysis}

Q10, C888, a physical mixture of C888:Q10, blank/ loaded SLN, and blank/loaded liposome formulations were analyzed. The samples to be tested were sealed in aluminum pans $(50 \mu \mathrm{L})$ and placed in differential scanning calorimeter (DSC 8000; PerkinElmer, Waltham, MA). DSC analysis was conducted under nitrogen flow $(20 \mathrm{~mL} / \mathrm{minute})$ in a temperature range between $30^{\circ} \mathrm{C}-300^{\circ} \mathrm{C}$.

\section{Cell culture studies Cytotoxicity studies}

Cells between the second and fifth passage of normal human dermal fibroblasts from juvenile foreskin (PromoCell $\mathrm{GmbH}$, Heidelberg, Germany) were employed for cytotoxicity studies. The growth media of fibroblasts consisted of Dulbecco's Modified Eagle Medium (DMEM; SigmaAldrich) augmented with 10\% foetal bovine serum (SigmaAldrich) with $200 \mathrm{IU} / \mathrm{mL}$ penicillin, and with $0.2 \mathrm{mg} / \mathrm{mL}$ streptomycin. The cells were kept in an incubator with an atmosphere of $5 \% \mathrm{CO}_{2}$ and $95 \%$ relative humidity at $37^{\circ} \mathrm{C}$.
Ninety-six-well plates (area of $0.34 \mathrm{~cm}^{2}$ ) were used to seed the fibroblasts at a density $10^{5}$ cells $/ \mathrm{cm}^{2}$ in each well. Cells were grown for 24 hours to obtain sub-confluence. After 24 hours the medium was removed and the samples to be tested were replaced. The cells were put in contact with $200 \mu \mathrm{L}$ of SLN-Q10 and LIPO-Q10 in the concentration range of $10-250 \mu \mathrm{M}$. After the incubation of cell substrates for 24 hours, the medium was removed and the (3-(4,5-Dimethylthiazol-2-yl)-2,5-diphenyltetrazolium bromide) (MTT) test was performed. $125 \mu \mathrm{L}$ of MTT solution (Sigma-Aldrich) at $0.25 \mu \mathrm{g} / \mathrm{mL}$ in Hank's buffered salt solution ( $\mathrm{pH} 7.4$ ) was put in contact with each cell substrate for 3 hours. An enzyme-linked immunosorbent assay plate reader (iMark ${ }^{\circledR}$ absorbance reader; Bio-Rad, Hercules, CA) at a wavelength of $570 \mathrm{~nm}$ with a reference wavelength of $655 \mathrm{~nm}$ was used to assay the absorbance. The \% ratio between the absorbance of each sample and the absorbance of cell substrate maintained in contact with the growth medium was expressed as cell viability. ${ }^{10}$

\section{Proliferation studies}

To evaluate the effect of oxidative stress on cell viability, additional cell culture studies were seeded in each well of 96-well plates and grown in the same conditions as for cytotoxicity studies. After 24 hours, the media in the wells were replaced with SLN and liposomes at two selected Q10 concentrations $(25 \mu \mathrm{M}$ and $50 \mu \mathrm{M})$. After 4 hours of contact, $1.5 \mathrm{mM} \mathrm{H}_{2} \mathrm{O}_{2}$ was added in these wells. This was the optimum $\mathrm{H}_{2} \mathrm{O}_{2}$ concentration causing oxidative damage avoiding cell death. After an additional 24 hours of contact, the viability was evaluated with an MTT test and the cell viability was determined as previously described. ${ }^{10}$

\section{Evaluation of intracellular ROS accumulation}

2,7-dichlorfluoresceine (DCFH-DA) acetate was employed as the fluorescent probe to evaluate intracellular accumulation of ROS. In this assay, cells are incubated with DCFH-DA and are able to cross the cell membrane. Once inside, the DCFH-DA loses its acetile groups and is no more able to go out of the cell. At this point it becomes nonfluorescent (DCFH). Only in the presence of ROS can it be oxidized in DCF, becoming fluorescent again. Higher fluorescence intensity means higher oxidative damage and then lower antioxidant activity.

Fibroblasts were seeded in 12-well plates (area of $3.8 \mathrm{~cm}^{2}$ ) at a density $10^{5}$ cells $/ \mathrm{cm}^{2}$. Cells were grown for 48 hours to obtain subconfluence. $50 \mu \mathrm{M}$ of SLN-Q10, LIPO-Q10, and Q10 alone, were put in contact with the cells. $1.5 \mathrm{mM}$ $\mathrm{H}_{2} \mathrm{O}_{2}$ was added in each well after 4 hours of contact. Cell 
substrates were incubated for a further 24 hours. Subsequently $1 \mathrm{mM}$ DCFH-DA was put in cell substrates for 15 minutes. To obtain a suspension, cells were scraped from the bottom of each well. This suspension was centrifuged at $1500 \mathrm{rpm}$ for 5 minutes. After the removal of surnatant, the cell pellet was re-suspended in $500 \mu \mathrm{L}$ phosphate buffered solution. A cytofluorometer (Navios Flow Cytometer; Beckman Coulter, Inc, Brea, CA) was used to assay these cell suspensions at the excitation wavelength of $488 \mathrm{~nm}$ and the emission wavelength of $525 \mathrm{~nm}$. The data were analyzed by Kaluza Analysis software (Beckman Coulter Inc). ${ }^{16}$

\section{Statistical analysis}

Statistical analysis was conducted by ANOVA followed by Tukey's test for comparisons between groups. The significance level was taken as $95 \%(P<0.05)$.

\section{Results and discussion}

The physical properties of nanosystems might affect in vivo behavior. In our previous study, it was reported that PS and ZP have important roles in penetration properties of lipid nanoparticles. ${ }^{9}$ The data in terms of PS, PI, ZP, and EE\% of SLNs and liposomes prepared with and without Q10 incorporation are shown in Table 1.

The PS obtained with SLNs, in either a blank or loaded state, were significantly smaller than the liposome formulations. The Q10 loading did not affect the PS of SLN system significantly. However, the addition of Q10 decreased the dimension of liposomes $(P<0.05)$. It is known that lipid composition has a significant impact on liposome size and drug loading. ${ }^{17}$ A decrement in the liposome dimension might be due to the shrinkage of the aqueous volume of the vesicle resulting in smaller dimensions by the addition of Q10, which is a very lipophilic substance $(\log \mathrm{P}>>10) .{ }^{18}$ This phenomenon could be explained by either the osmotic effect or the membrane folding. Since the total area of the membrane of each liposome was maintained, the folding of the membrane might cause this size reduction. In a study by Nomura et $\mathrm{al}^{19}$ the liposomes gradually shrank in size
( 5 times smaller) with the addition of peptides and the folding/piling up of liposomal membranes was confirmed by electron microscopy observations.

PI values of the formulations indicated that uniform colloidal systems could be formed for both liposomes and SLNs, with the methods applied in this study. Q10 addition reduced the overall charge of the SLNs as it was seen also in our previous studies conducted with resveratrol and cyclosporine A. ${ }^{10,14}$ On the contrary, Q10 addition increased the total electrical charge of liposomes. Similarly, in a study by Jukanti et al the electrical charge significantly increased from $-34.1 \pm 2.6 \mathrm{mV}$ up to $-46.9 \pm 2.7 \mathrm{mV}$ in case of Q10 addition in liposomes. ${ }^{20}$

The drug entrapment efficiency of Q10 was determined as $73.1 \%$ and $89.2 \%$ for liposomes and SLNs respectively, by a validated HPLC method. The enhanced encapsulation efficiency determined with SLNs in comparison to liposomes was an expected result, since it is one of the advantages of SLN over liposomes. Besides, the EE\% of Q10 in liposomes was also enough to say that Q10 could be encapsulated in liposomes successfully by film hydration method. Verma et al reported that film hydration was the most efficient method for Q10 encapsulation into liposomes in comparison to other methods. ${ }^{21}$ It is known that the partition of the drug between the lipid of membrane and the water, influences the $\mathrm{EE} \%$ of drugs in liposomes. ${ }^{22}$ Since Q10 is very lipophilic, its partition would be in favor of hydrophobic regions. Therefore, it was considered that Q10 might have been trapped in the double layers of phospholipids. TEM images acted as evidence for the colloidal size and homogenous structure of liposomes and SLNs (Figure 1).

DSC evaluation can give good clues about whether a drug is entrapped in the bilayer or in the aqueous compartment of the liposomes ${ }^{23}$ and helps to understand the melting and re-crystallization behavior of materials like lipid nanoparticles. ${ }^{24}$ DSC analysis was performed to examine the melting and crystallization properties of SLN and liposomes. The DSC thermograms of the formulations were given in Figures 2 and 3.

Table I PS, PI, ZP, and EE\% of blank and QI0-loaded SLNs and liposomes

\begin{tabular}{lllll}
\hline Formulation & PS \pm SD $(\mathbf{n m})$ & PI \pm SD & ZP \pm SD $(\mathbf{m V})$ & EE\% \pm SD \\
\hline Blank liposome & $367.9 \pm 7.5$ & $0.413 \pm 0.01$ & $-32.8 \pm 0.2$ & - \\
LIPO-QI0 & $301.1 \pm 8.2$ & $0.458 \pm 0.03$ & $-36.6 \pm 0.9$ & $73.1 \pm 1.7$ \\
Blank SLN & $164.1 \pm 9.4$ & $0.294 \pm 0.05$ & $-18.64 \pm 1.2$ & - \\
SLN-QI0 & $152.4 \pm 7.9$ & $0.272 \pm 0.03$ & $-13.67 \pm 1.3$ & $89.2 \pm 3.8$ \\
\hline
\end{tabular}

Note: The results are the means \pm SD $(n=6)$.

Abbreviations: PS, particle size; PI, polydispersity index; ZP, zeta potential; EE\%, entrapment efficiency; QI0, coenzyme QI0; SLN, solid lipid nanoparticle; SD, standard deviation; LIPO-QI0, QI0-loaded liposomes; SLN-QI0, QI0-loaded solid lipid nanoparticles. 

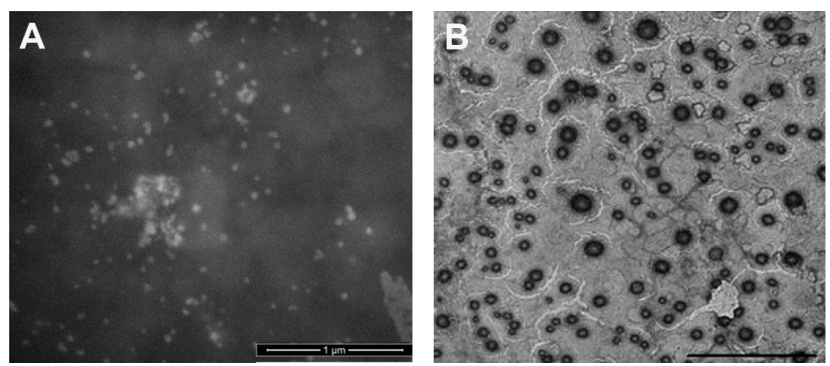

Figure I TEM images of (A) SLN-QIO and (B) LIPO-QIO.

Notes: (A) The bar is I $\mu \mathrm{m}$. (B) The bar is $5 \mu \mathrm{m}$.

Abbreviations: TEM, transmission electron microscopy; SLN-QI0, QI0-loaded solid lipid nanoparticles; LIPO-Q10, Q10-loaded liposomes.

The melting points for $\mathrm{C} 888$ and Q10 were determined as $72.31^{\circ} \mathrm{C}$ and $53.96^{\circ} \mathrm{C}$, respectively. It was observed that C888 was crystallized during the cooling process of SLN production (Figure 2). The melting peak of Q10 could not be observed in SLN formulation, due to its solubility in the lipid phase (Figure 2). In the DSC thermogram of Q10-loaded liposomal dispersion (Figure 3), the Q10 peak was also lost, indicating good interaction of all components. Since Q10 is highly lipophilic it is possible that it might be entrapped in the bilayer compartment of liposome.

It is known that the structure of liposomes and SLNs may be destroyed by several enzymatic mechanisms such as lipases. ${ }^{14}$ However, these systems protect labile drugs from degradation by encapsulation and increase their permeation. Zhou et $\mathrm{al}^{25}$ prepared Q10-loaded lipid nanocapsules with nile red and observed the accumulation through skin layers by the intensity of fluorescence. It was determined that lipid nanocapsules could be targeted to both epidermis and dermis. In addition, the in vitro assessment of the cytotoxicity on human dermal fibroblasts has been used as an approach to evaluate the toxicity of the different formulations. ${ }^{26}$

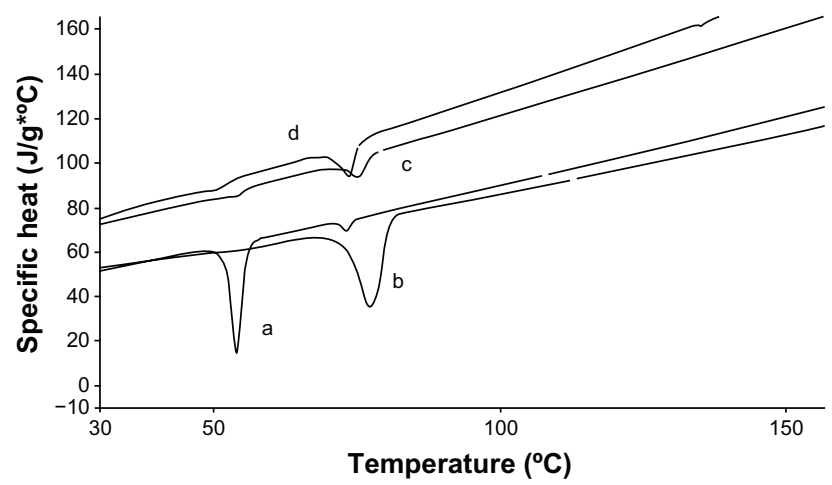

Figure 2 DSC thermograms of (a) QIO; (b) compritol; (c) blank SLN; and (d) SLN-QI0.

Abbreviations: DSC, differential scanning calorimetry; QI0, coenzyme QI0; SLN, solid lipid nanoparticle; SLN-Q10, Q I0-loaded solid lipid nanoparticles.

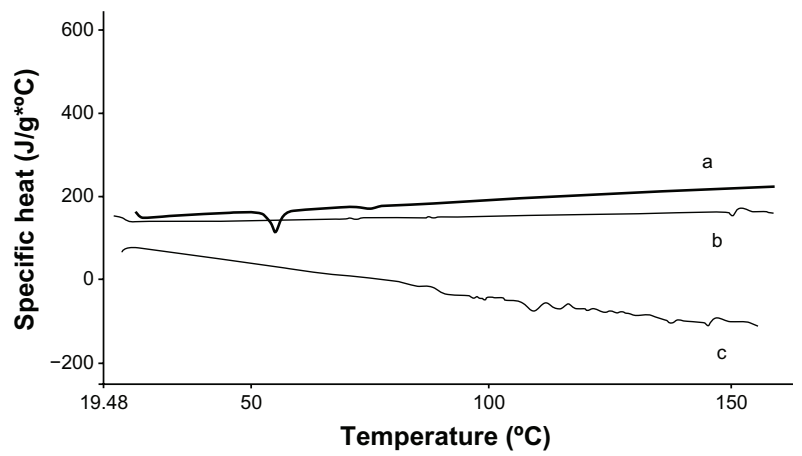

Figure 3 DSC thermograms of (a) QI0 and (b) blank liposome and (c) LIPO-QI0. Abbreviations: DSC, differential scanning calorimetry; QIO, coenzyme QIO; LIPO-QI0, QI0-loaded liposomes.

The delivery of Q10 to fibroblasts is particularly important because oxidative stress affects several pathways especially in dermal fibroblasts. A major cause of oxidative stress in skin is solar UV radiation. This phenomenon influences pathways in ways that closely mimic ROS. ${ }^{27}$ Recently, Reelfs et al have shown that labile iron in skin fibroblast, which is involved in the activation of a number of transcription factors, was released by UVA irradiation. ${ }^{28}$ It is also known that ${ }^{1} \mathrm{O}_{2}$ can initiate c-jun $\mathrm{N}$-terminal kinase signaling, which leads to interstitial collagenase induction as well as the synthesis of proinflammatory cytokines in UVA-irradiated fibroblasts. ${ }^{27}$ It was also proven that aging skin fibroblasts have decreased catalase activity. Accumulation of ROS owing to catalase attenuation may be a critical aspect of the MAPK signaling changes that result in skin aging and photoaging in human skin in vivo. ${ }^{29}$ Therefore, it was thought that evaluation of the effective delivery of Q10 in dermal fibroblasts will be beneficial due to its involvement in several pathways that is found in fibroblasts. Thus cell culture studies were conducted on these cells.

Our cell culture studies revealed that at $250 \mu \mathrm{M}$, cellular toxicity was observed for both formulations $(P<0.05)$. At the level of $100 \mu \mathrm{M}$, a significant difference occurred between SLN and liposome treated groups. In the SLN exposed group the vitality of the cells were over $80 \%$, however for liposome treated group, dramatic cell death was observed (Figure 4). The critical concentration of Q10 in the nanosystems that the cells can survive was determined as $50 \mu \mathrm{M}$ for both formulations. At this concentration, in the liposome-treated group, cell proliferation was observed (viability over 120\%). At the lowest Q10 concentration of $10 \mu \mathrm{M}$, no difference could be seen in terms of cell viability between SLNs and liposome treated groups. However at $25 \mu \mathrm{M}$ the viability results were again in favor of liposomes (Figure 4). Thus it was decided to carry out additional cell 


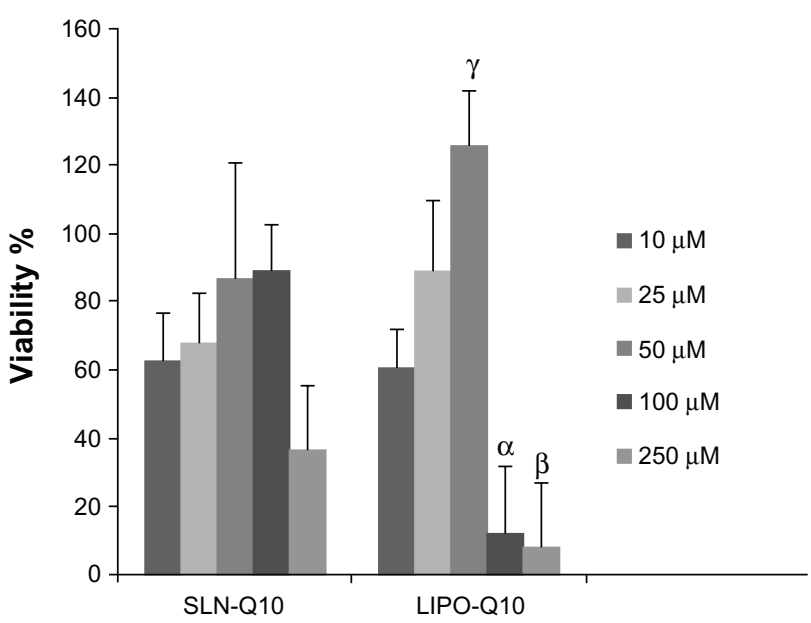

Figure 4 Viability (\%) of fibroblast cells after 24 hours of treatment with I0, 25, 50, 100 , and $250 \mu \mathrm{M}$ of SLNs and liposomes.

Notes: The results are the means \pm SD. ${ }^{\alpha, \beta, \gamma p}<0.05$; viability $\%$ of fibroblasts; SLNs vs liposomes.

Abbreviations: SLN, solid lipid nanoparticle; SD, standard deviation; SLN-QI0, Q I0-loaded solid lipid nanoparticles; LIPO-QI0, Q I0-loaded liposomes.

viability studies under oxidative conditions at the concentration levels of $25 \mu \mathrm{M}$ and $50 \mu \mathrm{M}$.

Under the oxidative conditions, it was seen that both $25 \mu \mathrm{M}$ and $50 \mu \mathrm{M}$ of Q10 concentrations were not able to protect cells from oxidative damage when encapsulated in SLNs. Liposomes performed better protective effects and $50 \mu \mathrm{M}$ was a better choice of concentration for this aim. The proliferation of the cells led to a viability value, such as $120 \%$. SLN's protective effect was lower when compared to Q10 alone (Figure 5). To understand the underlying mechanism of this result, cytofluorometry studies were conducted at $50 \mu \mathrm{M}$.

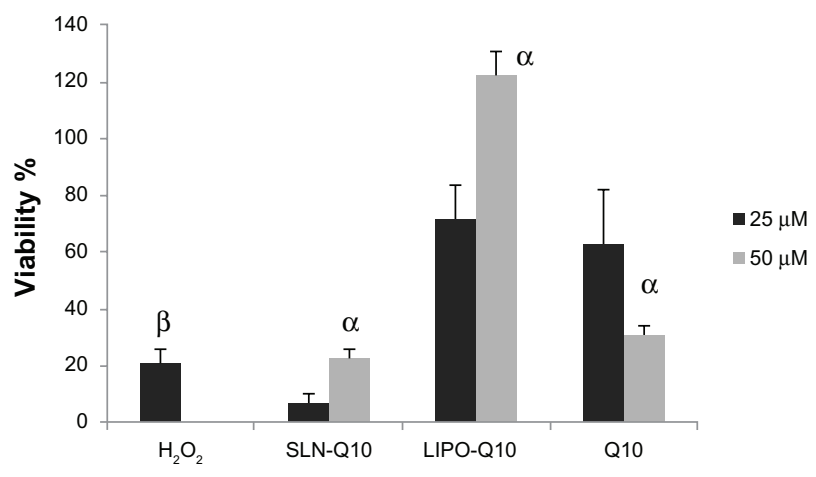

Figure 5 Viability (\%) of fibroblast cells treated with $25 \mu \mathrm{M}$ and $50 \mu \mathrm{M}$ of SLNs and liposomes after contact with $\mathrm{H}_{2} \mathrm{O}_{2}$ at concentration of $2 \mathrm{mM}$.

Notes: The results are the means \pm SD. ${ }^{\alpha} p<0.05$; viability $\%$ of fibroblasts determined for $50 \mu \mathrm{M}$ vs $25 \mu \mathrm{M}$; ${ }^{\beta} \mathrm{P}<0.05$; viability \% of fibroblasts treated with only $\mathrm{H}_{2} \mathrm{O}_{2}$.

Abbreviations: SLN, solid lipid nanoparticle; SD, standard deviations; SLN-QI0, Q10-loaded solid lipid nanoparticles; LIPO-QI0, QI0-loaded liposomes; QI0, coenzyme QI0.
The number of cells versus fluorimetric intensity is presented in Figure 6. $1 \mathrm{mM}$ of DCFH-DA was used to differentiate ROS fluorescence and noise. ROS production in the cells comes as an output of fluorescence. In the case of this study, if the fluorescence intensity gets higher, it means that the antioxidant effect of the tested formulation is lower.

Figure 7 presents the histograms of cells versus fluorimetric intensity evaluated for Q10, SLN-Q10, LIPO-Q10, control, and $\mathrm{H}_{2} \mathrm{O}_{2}$ treated samples. The viability of cell substrates treated with SLN and liposome samples was confirmed with the morphologic parameters (Figure 7), to indicate that the oxidative damage did not cause cell death.

The oxidative damage caused by $\mathrm{H}_{2} \mathrm{O}_{2}$ treatment is directly related fluorescence intensity due to ROS production. The fluorescence intensity gets higher as the intracellular ROS concentration gets higher. The highest ROS accumulation was determined with $\mathrm{H}_{2} \mathrm{O}_{2}$ treated cell substrate, as indicated by cell peak characterized by fluorescence intensity ranging from 10 to 100 . The mean fluorescence intensity $\%$ result obtained from untreated cell substrate was $39.55 \%$. The results obtained from formulation treated and $\mathrm{H}_{2} \mathrm{O}_{2}$ treated groups could be listed in order as:

$$
\mathrm{H}_{2} \mathrm{O}_{2(49.27)}>\mathrm{Q} 10_{(44.86)} \geq \mathrm{SLN}-\mathrm{Q} 10_{(44.34)}>{\mathrm{LIPO}-\mathrm{Q} 10_{(27.41)}}
$$

In the peroxide-treated cells the fluorescence reaches an order of magnitude higher than the control to indicate a higher oxidative damage in absence of an antioxidant. The liposometreated cell substrate was characterized by a significantly low ROS accumulation. The cell substrates treated with SLN-Q10 were characterized by cell fluorescence intensity almost similar to the substrate treated with only Q10. This result was surprising because of the expected advantage of SLNs as an enhancer in cellular uptake due to nanosize. In our previous study, resveratrol (RSV)-loaded NLC showed fluorescence below that of RSV and RSV-loaded SLN, indicating less ROS production, and this result was attributed to smaller dimensions of RSV-loaded NLC with a reduced negative electrical charge. ${ }^{10}$ However, in this study, the dimension of LIPO-Q10 was nearly 2-fold that of SLN-Q10 with the magnitude of ZP higher than SLN-Q10.

It was thought that, since Q10 was highly lipophilic (practically insoluble in water), a delayed release might be responsible for this result. In the studies of Farboud et $\mathrm{al}^{30}$ and Teeranachaideekul et $\mathrm{al}^{6}$ it was reported that Q10 was released slowly from the solid matrix of the lipid. In another study, no Q10 release could be seen during 7 days when 

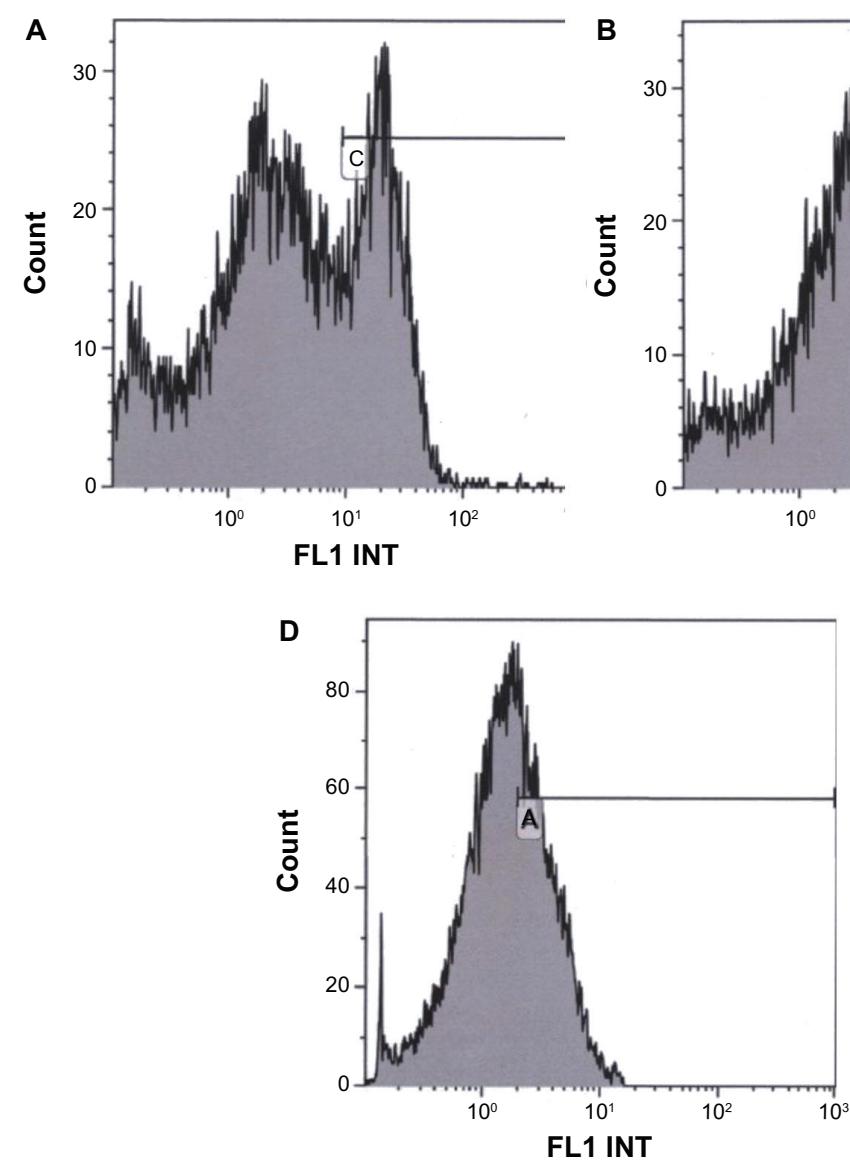

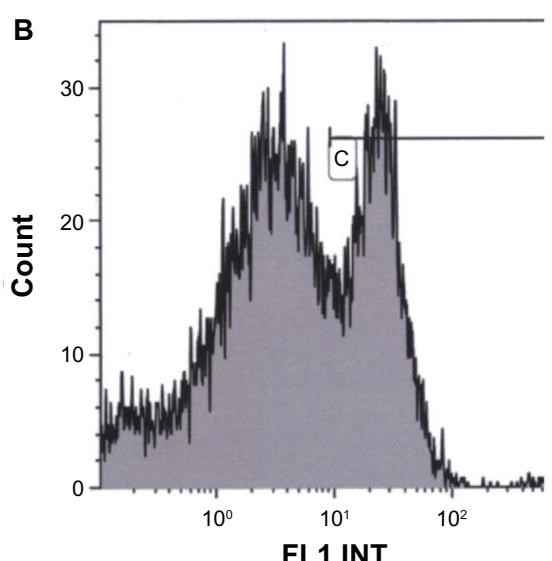

FL1 INT

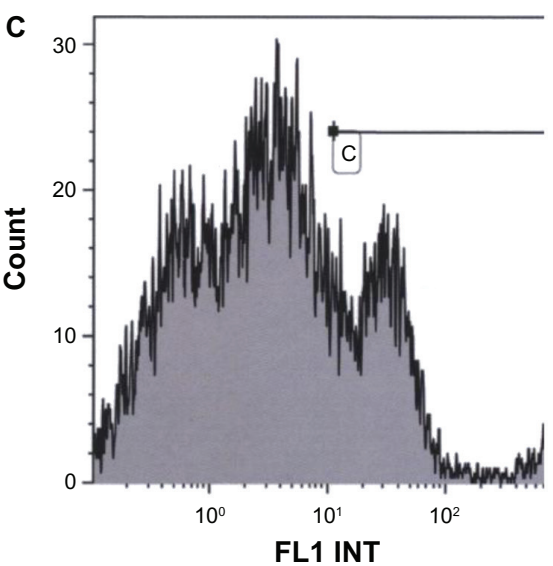

E

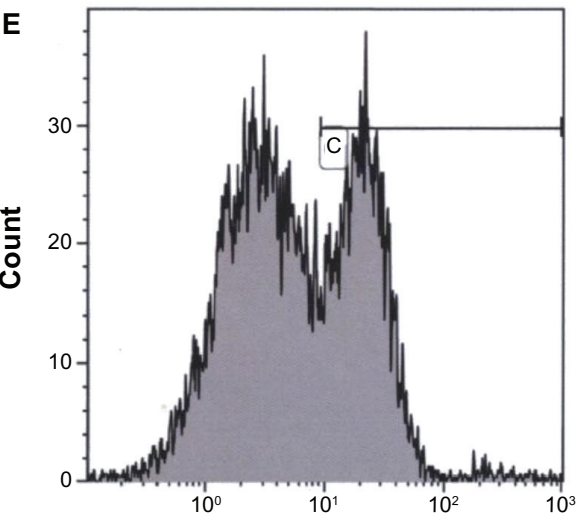

FL1 INT

Figure 6 Signal intensity histograms of cells incubated with (A) QIO, (B) SLN-QI0, and (C) LIPO-QI0; (D) control; (E) $\mathrm{H}_{2} \mathrm{O}_{2}$-treated samples. Abbreviations: FLI INT, fluorimetric intensity; Q I0, coenzyme Q I0; SLN-Q I0, Q I0-loaded solid lipid nanoparticles; LIPO-Q I0, Q I0-loaded liposomes.
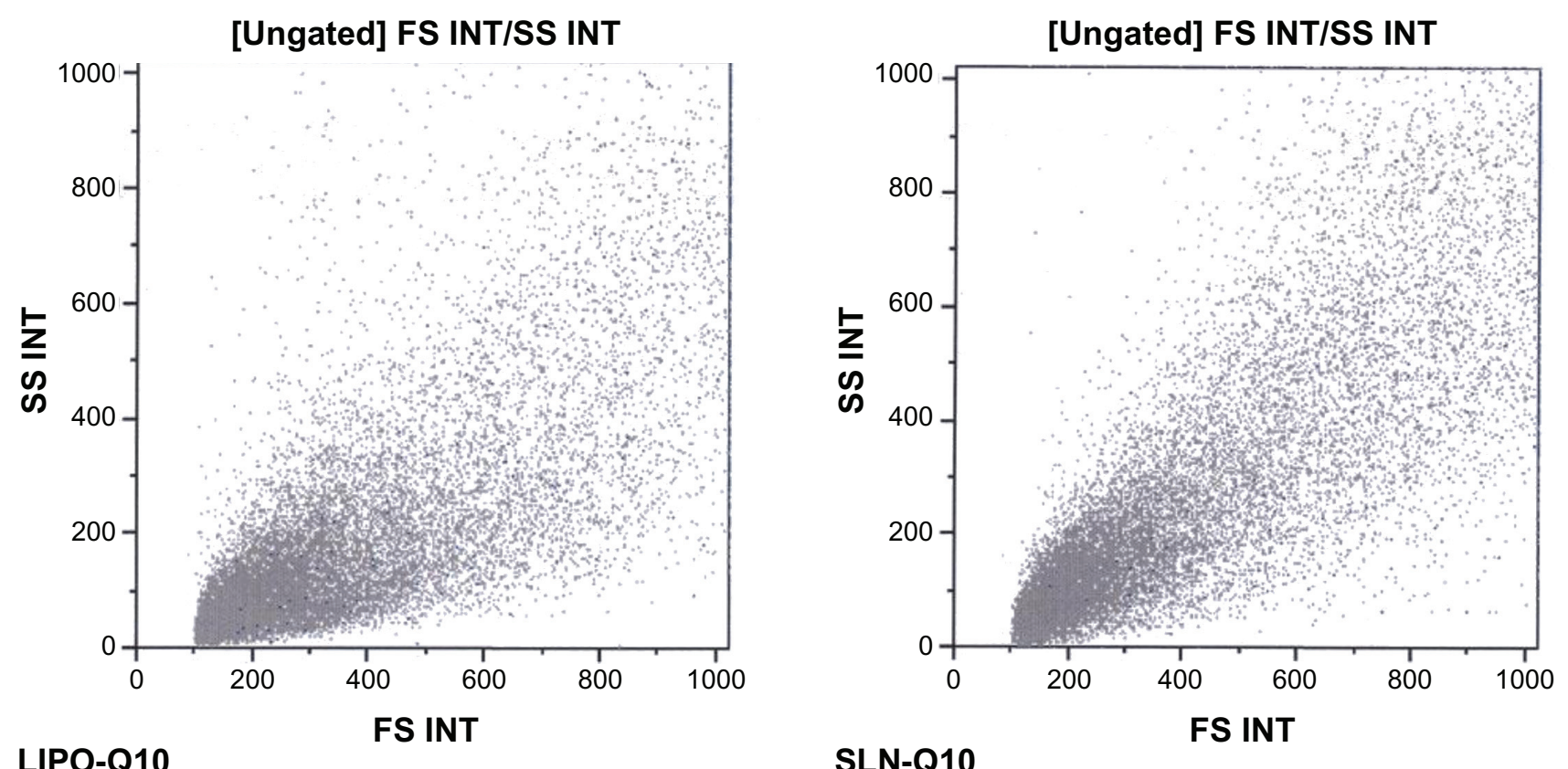

Figure 7 Morphologic parameter of fibroblasts treated with SLN and liposome samples.

Abbreviations: SLN, solid lipid nanoparticle; SLN-QI0, QIO-loaded solid lipid nanoparticles; LIPO-QI0, QI0-loaded liposomes; FS INT, forward scatter; SS INT, side scatter. 
the nanoparticles were suspended in water. ${ }^{31}$ On the other hand, liposomes may interact with the cell membranes and disorder the membrane properties. ${ }^{32}$ It was shown that, the permeability barrier of the stratum corneum was weakened by phosphatidylcholine. ${ }^{33}$ Makabi-Panzu et a ${ }^{34}$ reported that incorporation of Q10 in liposomes enhanced the cellular uptake by macrophage cells. Therefore it was thought that the superior antioxidant effect of LIPO-Q10 observed in this study might be due to membrane perturbing properties of liposomes such as adsorption and fusion. ${ }^{35}$

\section{Conclusion}

Nanosystems made from different structures were previously shown to enhance dermal uptake or improve tolerability of active substances. In this study, SLN-Q10 and LIPO-Q10 were prepared by means of high shear homogenization and thin film hydration method, respectively. The PS of SLNs obtained was significantly smaller than liposomes. However the overall charge was lower in comparison to liposomes. Q10 was not in a crystalline state for both of the nanosystems, indicating its solubility in lipid phases.

After cell culture tests conducted under normal and oxidative conditions, $50 \mu \mathrm{M}$ was considered the efficient Q10 concentration for cell viability. LIPO-Q10 performed better cell proliferation-inducing properties and ROS production inside the cells was decreased by nearly $50 \%$ in comparison to the negative control.

As a conclusion, in terms of dermal antioxidant activity of Q10, liposomes were regarded as better and more promising delivery systems in comparison to SLNs.

\section{Acknowledgment}

We wish to thank Dr G Viarengo and Dr M Cervio from the hospital IRCCS POLICLINICO SAN MATTEO S.C. SIMT - Servizio di Immunoematologia e Medicina Trasfusionale for the measurements of cytofluorometry.

\section{Disclosure}

The authors report no conflicts of interest in this work.

\section{References}

1. Hoppe U, Bergemann J, Diembeck W, et al. Coenzyme Q10, a cutaneous antioxidant and energizer. Biofactors. 1999;9(2-4):371-378.

2. Kim DW, Hwang IK, Kim DW, et al. Coenzyme Q10 effects on manganese superoxide dismutase and glutathione peroxidase in the hairless mouse skin induced by ultraviolet B irradiation. Biofactors. 2007;30(3):139-147.

3. Black HS, de Gruijl FR, Forbes PD, et al. Photocarcinogenesis: an overview. J Photochem Photobiol B. 1997;40(1):29-47.

4. Lenaz G, Fato R, Formiggini G, Genova ML. The role of Coenzyme Q in mitochondrial electron transport. Mitochondrion. 2007;7(Suppl):S8-S33.
5. Muta-Takada K, Terada T, Yamanishi H, et al. Coenzyme Q10 protects against oxidative stress-induced cell death and enhances the synthesis of basement membrane components in dermal and epidermal cells. Biofactors. 2009;35(5):435-441.

6. Teeranachaideekul V, Souto EB, Junyaprasert VB, Müller RH. Cetylpalmitate-based NLC for topical delivery of Coenzyme Q(10) development, physicochemical characterization and in vitro release studies. Eur J Pharm Biopharm. 2007;67(1):141-148.

7. Liu J, Hu W, Chen H, Ni Q, Xu H, Yang X. Isotretinoin-loaded solid lipid nanoparticles with skin targeting for topical delivery. Int J Pharm. 2007;328(2):191-195.

8. Schreier H, Bouwstra J. Liposomes and niosomes as topical drug carriers: dermal and transdermal drug delivery. $J$ Control Release. 1994;30(1):1-15.

9. Souto EB, Wissing SA, Barbosa CM, Müller RH. Development of a controlled release formulation based on SLN and NLC for topical clotrimazole delivery. Int J Pharm. 2004;278(1):71-77.

10. Gokce EH, Korkmaz E, Dellera E, Sandri G, Bonferoni MC, Ozer O. Resveratrol-loaded solid lipid nanoparticles versus nanostructured lipid carriers: evaluation of antioxidant potential for dermal applications. Int J Nanomedicine. 2012;7:1841-1850.

11. Dingler A, Blum RP, Niehus H, Müller RH, Gohla S. Solid lipid nanoparticles (SLN/Lipopearls) - a pharmaceutical and cosmetic carrier for the application of vitamin $\mathrm{E}$ in dermal products. J Microencapsul. 1999;16(6):751-767.

12. Junyaprasert VB, Teeranachaideekul V, Souto EB, Boonmed P, Müller RH. Q10-loaded NLC versus nanoemulsions: Stability, rheology and in vitro skin permeation. Int J Pharm. 2009;377(1-2): 207-214.

13. Mura P, Maestrelli F, González-Rodríguez ML, Michelacci I, Ghelardini C, Rabasco AM. Development, characterization and in vivo evaluation of benzocaine-loaded liposomes. Eur J Pharm Biopharm. 2007;67(1):86-95.

14. Gokce EH, Sandri G, Bonferoni MC, et al. Cyclosporine A loaded SLNs: evaluation of cellular uptake and corneal cytotoxicity. Int $J$ Pharm. 2008;364(1):76-86.

15. Karpińska J, Mikołuć B, Motkowski R, Piotrowska-Jastrzebska J. HPLC method for simultaneous determination of retinol, alphatocopherol and coenzyme Q10 in human plasma. $J$ Pharm Biomed Anal. 2006;18(42):232-236.

16. Afzal M, Matsugo B, Aoyama K, Takeuchi T. Method to overcome photoreaction, a serious drawback to the use of dichlorofluorescin in evaluation of reactive oxygen species. Biochem Biophys Res Commun. 2003;304(4):619-624.

17. Ramana LN, Sethuraman S, Ranga U, Krishnan UM. Development of a liposomal nanodelivery system for nevirapine. J Biomed Sci. 2010;17:57.

18. Gopta OA, Semenov AY, Bloch DA. Electrogenic proton transfer in Rhodobacter sphaeroides reaction centers: effect of coenzyme $Q(10)$ substitution by decylubiquinone in the $\mathrm{Q}(\mathrm{B})$ binding site. FEBS Lett. 2001;499(1-2):116-120.

19. Nomura F, Inaba T, Ishikawa $\mathrm{S}$, et al. Microscopic observations reveal that fusogenic peptides induce liposome shrinkage prior to membrane fusion. Proc Natl Acad Sci U S A. 2004;101(10):3420-3425.

20. Jukanti R, Devaraj G, Devaraj R, Apte S. Drug targeting to inflammation: studies on antioxidant surface loaded diclofenac liposomes. Int J Pharm. 2011;414(1-2):179-185.

21. Verma DD, Hartner WC, Thakkar V, Levchenko TS, Torchilin VP. Protective effect of coenzyme Q10-loaded liposomes on the myocardium in rabbits with an acute experimental myocardial infarction. Pharm Res. 2007;24(11):2131-2137.

22. Nii T, Ishii F. Encapsulation efficiency of water-soluble and insoluble drugs in liposomes prepared by the microencapsulation vesicle method. Int J Pharm. 2005;298(1):198-205.

23. Weiner M, Martin F, Riaz M. Liposomes as a drug delivery system. Drug Dev Ind Pharm. 1989;15(10):1523-1554.

24. Timms RE. Fractional crystallization - the fat modification process for the 21st century. Eur J Lipid Sci Technol. 2005;107(1):48-57. 
25. Zhou H, Yue Y, Liu G, et al. Characterisation and Skin Distribution of Lecithin-Based Coenzyme Q10-Loaded Lipid Nanocapsules. Nanoscale Res Lett. 2010;5(10):1561-1569.

26. Schubert MA, Müller-Goymann CC. Characterisation of surfacemodified solid lipid nanoparticles (SLN): influence of lecithin and nonionic emulsifier. Eur J Pharm Biopharm. 2005;61(1-2):77-86.

27. Bickers DR, Athar M. Oxidative stress in the pathogenesis of skin disease. J Invest Dermatol. 2006;126(12):2565-2575.

28. Reelfs O, Tyrrell RM, Pourzand C. Ultraviolet a radiation-induced immediate iron release is a key modulator of the activation of NF-kappaB in human skin fibroblasts. J Invest Dermatol. 2004;122(6):1440-1447.

29. Fisher GJ, Kang S, Varani J, et al. Mechanisms of photoaging and chronological skin aging. Arch Dermatol. 2002;138(11):1462-1470.

30. Farboud ES, Nasrollahi SA, Tabbakhi Z. Novel formulation and evaluation of a Q10-loaded solid lipid nanoparticle cream: in vitro and in vivo studies. Int J Nanomedicine. 2011;6:611-617.
31. Hsu CH, Cui Z, Mumper RJ, Jay M. Preparation and characterization of novel coenzyme Q10 nanoparticles engineered from microemulsion precursors. AAPS Pharm Sci Tech. 2003;4(3):E32.

32. Fang JY, Hong CT, Chiu WT, Wang YY. Effect of liposomes and niosomes on skin permeation of enoxacin. Int J Pharm. 2001; 219(1-2):61-72.

33. Kato A, Ishibashi Y, Miyake Y. Effect of egg yolk lecithin on transdermal delivery of bunazosin hydrochloride. J Pharm Pharmacol. 1987;39(5):399-400.

34. Makabi-Panzu B, Sprott GD, Patel GB. Coenzyme Q10 in vesicles composed of archaeal ether lipids or conventional lipids enhances the immuno-adjuvanticity to encapsulated protein. Vaccine. 1998;16(16): 1504-1510.

35. Lee WC, Tsai TH. Preparation and characterization of liposomal coenzyme Q10 for in vivo topical application. Int J Pharm. 2010; 395(1-2):78-83.
International Journal of Nanomedicine

\section{Publish your work in this journal}

The International Journal of Nanomedicine is an international, peerreviewed journal focusing on the application of nanotechnology in diagnostics, therapeutics, and drug delivery systems throughout the biomedical field. This journal is indexed on PubMed Central,

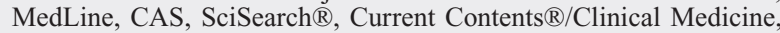

\section{Dovepress}

Journal Citation Reports/Science Edition, EMBase, Scopus and the Elsevier Bibliographic databases. The manuscript management system is completely online and includes a very quick and fair peer-review system, which is all easy to use. Visit http://www.dovepress.com/ testimonials.php to read real quotes from published authors.

Submit your manuscript here: http://www.dovepress.com/international-journal-of-nanomedicine-journal 